\title{
Coordinate Control Strategy for Current Stabilization in an Aluminum Smelter Including on Load Tap Changer
}

\author{
Heping Xü ${ }^{1}$,ipin $\mathrm{Xu}^{1}$, Yuan Gao ${ }^{2}$, Haoming Liu ${ }^{2}$ \\ ${ }^{1}$ NARI Technology Development Co. Ltd., Nanjing, China \\ ${ }^{2}$ College of Energy and Electrical Engineering, Hohai University, Nanjing, China \\ Email: xuheping@sgepri.sgcc.com.cn, liuhaom@hhu.edu.cn
}

Received March, 2013

\begin{abstract}
The performance of the current stabilization control system in an aluminum smelter affects the quality and the quantity of the electrolytic products. This paper elaborates the power supply, in which the diode rectifiers and the self-saturable reactors could keep the series current stable, then describes the basic principle of the rectifier unit control and the series current control. A coordinate strategy is proposed to keep the series current stable, the self-saturable reactors are controlled by a proportional-integral control scheme and the on load tap changers of transformer are triggered by the errors between the setting value of the series voltage and the measured values of the series voltage. Simulation results on PSCAD/EMTDC show that the effectiveness of the proposed strategy to keep the series current stable.
\end{abstract}

Keywords: Current Stabilization; Diode Rectifier; Self-saturable Reactor; OLTC; Coordinate Control

\section{Introduction}

The aluminum smelter is one of the important industrial loads. The dc current should be kept stable to ensure the efficient production. Not only the voltage's fluctuation on the grid side, but the anode effect in the aluminum smelter can cause the series current unstable. If the current control doesn't work well, the heat of the smelter might be unbalance, and the power consumption to product the aluminum might increase, both they would affect the quantity and the quality of the production to a great extent [1].

Most of the related papers focus on the concept or the practical application of the current stabilization control system of the aluminum electrolysis. While few of them touches the current stabilization control strategy based on the self-saturable reactor (SR) and the on load tap changer (OLTC) together. The basic structures of the current stabilization control system are discussed in [2-4]. The reasons of the changes of the series current caused by the smelters load changing, the voltage fluctuation on the grid side and the anode effect happen are investigated respectively in [5]. A proportional- integral (PI) control method is introduced to adjust the inductance of the self-saturable reactor in [6].

This paper describes the basic structure of the power supply in the aluminum smelter, and the basic principle of the rectifier unit control and the series current control at first. Then a coordinate control strategy is proposed in order to keep the series current stable, the PI control method is introduced into the self-saturable reactor, and the OLTC will be triggered by the big errors between the setting value of the series current and the measured series current. The proposed coordinate control strategy is carried out in PSCAD/EMTDC to validate the effectiveness.

\section{Current Stabilization Control Scheme}

In the aluminum smelter, the whole rectification system is comprised of several separated rectifier units in parallel. Each unit consists of the OLTC transformer, phase- shifting rectifier transformer, cophase counter parallel connection rectifiers, and some related auxiliary facilities. In general, the cophase counter parallel connection rectifier is of a 12 pulse circuit, including two 6-pluse's rectifier cubicles. The power supply schematic diagram of an aluminum smelter is shown in Figure 1, it is comprised of $4 \mathrm{dc}$ rectifier units. Each unit consists of the diode rectifiers and the self-saturable in series.

The " $\mathrm{N}+1$ " principle is adopted in the current stabilization control system. Here, 1 represents the master control, $\mathrm{N}$ represents the number of the rectifier units, e.g., $\mathrm{N}$ equals to 4 in Figure 1. The power of the electrolytic series is supplied from the $\mathrm{N}$ rectifier units, and the current of the electrolytic series is equal to the sum of all the rectifier units current. The master control 
ensures the series current stable.

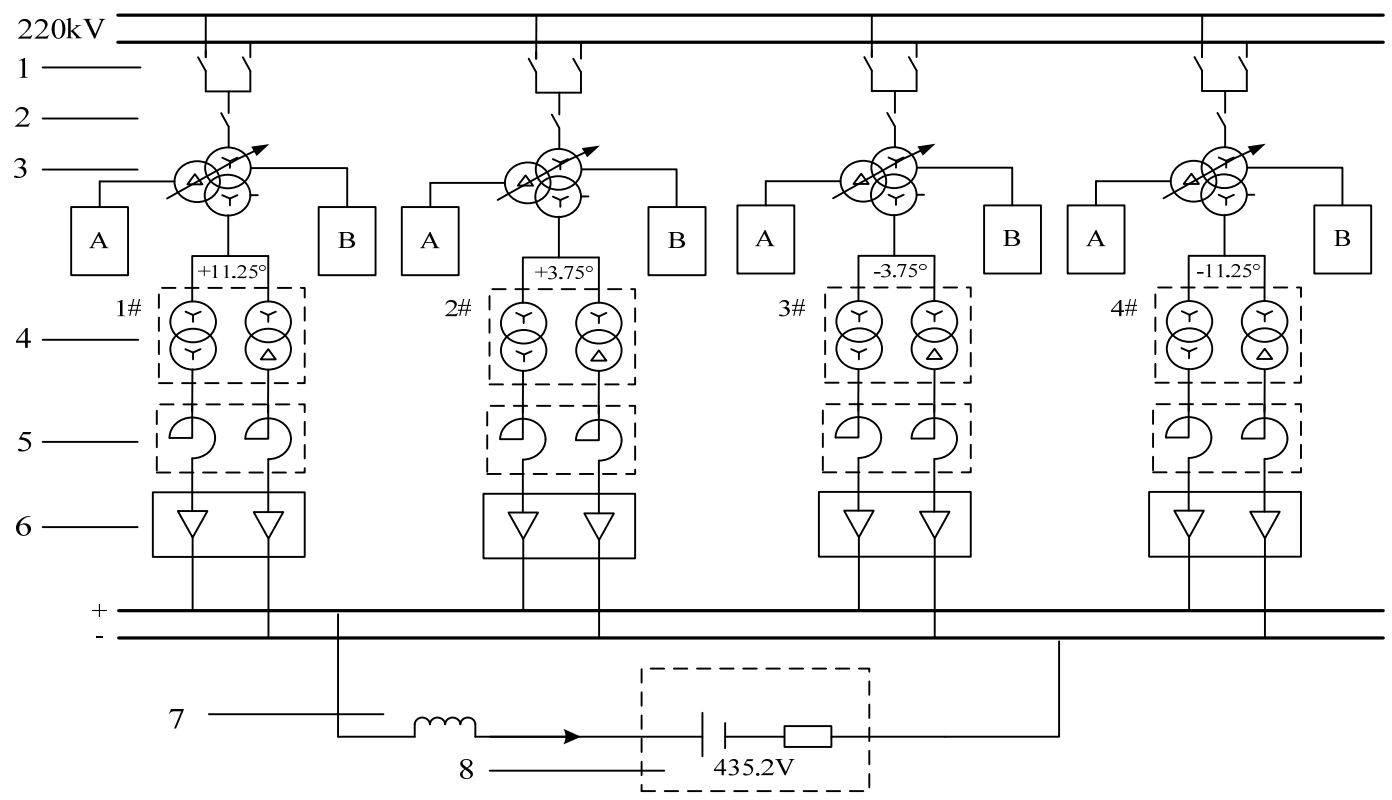

A - Filter Compensation Device; B - Grounding Equipments at the Neutral; 1 - Isolating Switch; 2 - Contactor Switch; 3 - OLTC Transformer; 4 Phase-shifting Rectifier Transformer; 5 - SR; 6 - cophase counter parallel connection rectifiers; 7 - Choke Inductor; 8 - The Equivalent Model of the Electrolytic Series

Figure 1. The power supply schematic diagram of an aluminum smelter.

\subsection{Current Stabilization Control of the Rectifier Unit}

The current stabilization control of the rectifier unit is realized through means of regulation on the self-saturable reactor, so as to keep the total load current stable, and ensure the balance of the currents between the two 6-pluse's rectifier cubicles.

The self-saturable reactor is made with a ferromagnetic material with nonlinear magnetization curve and with nonlinear saturation characteristics. It is composed of offset winding, control winding, and work winding. SR is connected in series into the rectifier bridge arm of the unit.

When an anode effect occurs, the series current $I$ decreases, and the dc currents $I_{d c}$ of every the rectifier units decrease. The error $\Delta I$ between the dc outputting current and the reference value of the rectifier unit increases, the duty cycle of the diode is adjusted after the PI control. In which, the conduction lag time of the diode is regulated via the work winding of the self-saturable reactor $[7,8]$. Finally, the dc outputting current can be adjusted close to the reference value. The whole control process of the current stabilization of the rectifier unit is shown in Figure 2.

\subsection{Series Current Stabilization Control}

The series current stabilization controller adjusts the selfsaturable reactors and the OLTC to ensure the total current stable. It depends on the amount of the error between the measured value of the outputting series current and the pre-setting value, and determines whether or not the OLTC need act. If needed, the taps of OLTC is regulated to a new appropriate position, and then the current stabilization control of the rectifier unit is activating to ensure the current stable [9, 10].

\section{Coordinate Control Strategy}

\subsection{Control Strategy of the SR}

The flux density curve of the SR is

$$
\frac{d B}{d H}=e^{a} H^{c} e^{\frac{b}{H}}
$$

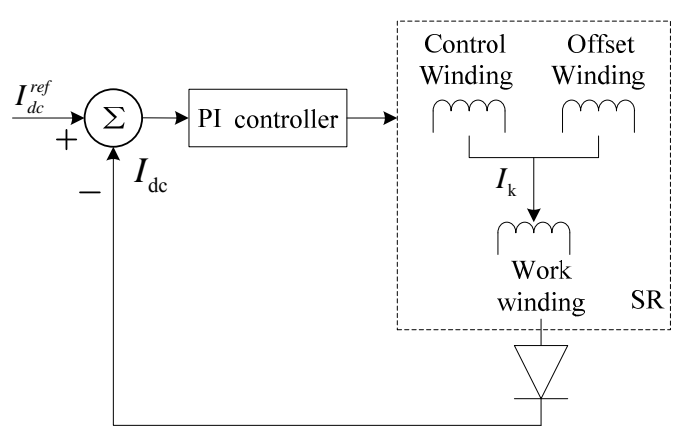

Figure 2. Control process of a rectifier unit.

where, $a, b$ and $c$ are the magnetization curve constants of SR, $B$ is magnetic flux density, $H$ is magnetic field 
density.

Taking no account of the magnetic leakage and iron loss, the reactance of SR is

$$
L=\frac{A n_{a c}^{2}}{l} \times \frac{d B}{d H}=\frac{A n_{a c}^{2}}{l} \times e^{a} H^{c} e^{\frac{b}{H}}
$$

where, $\mathrm{A}$ is the area of the section of the core $\left(\mathrm{cm}^{2}\right), n_{a c}$ is the number of the SR's work winding, $l$ is the average length of the magnetic circuit $(\mathrm{cm})$ [11].

For simplify, the SR is considered as a controllable nonlinear reactor, and it keeps constant if the system is steady. All setting values of the current of rectifier units are supposed as equal, the sum of them is the setting value of the electrolytic series current, that is, $I^{\text {ref }}=$ $N \cdot I_{d c}^{r e f}$. The error between $I_{d c}^{r e f}$ and $I_{d c}$, the measured value of the series current, is as the input of the PI controller, and the output from PI controller is added to the setting value of SR's inductance $L^{\text {ref }}$, the sum is the actual inductance after the regulation of SR.

The control diagram of the SR is shown in Figure 3. $K_{p}$ and $T$ are the proportional gain and integral time constant of PI controller.

Due to the magnetic characteristics of the SR, the regulating range is limited. It makes the regulating range of the outputting dc voltage on the load bus limited. In general, the regulator depth is about $70 \mathrm{~V}$, and the regulation could be carried out continuously.

\subsection{Control Strategy of OLTC}

In this paper, the voltage range of fineness regulation is $[0, \Delta \mathrm{Umax}]$, the range is divided into three sections. The first section is $[0, \mathrm{U} 1]$, called up shift section; the second section is [U1, U2], called unrestrained section; the third is [U2, $\Delta \mathrm{Umax}]$, called downshift section. The voltage range of unrestrained section is almost 1.5 times as the voltage change one step of OLTC caused, and the voltage range of up shift or downshift section can meet the requirements of the common fluctuation. For example, the voltage range of downshift section should cover the voltage change an anode effect caused.

The flow chart of the coordinate current stabilization control in an aluminum smelter is shown in Figure 4. If there exists a unit failure, unit maintenance or an anode effect, the series current varies a few from the reference value, and it exceeds the capability of the fineness regulation of SR, the upshift or downshift of OLTC should be

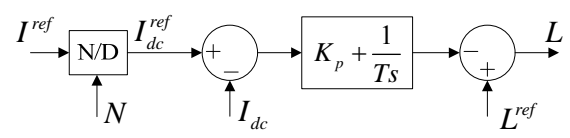

Figure 3. Control diagram of the self-saturable reactor.

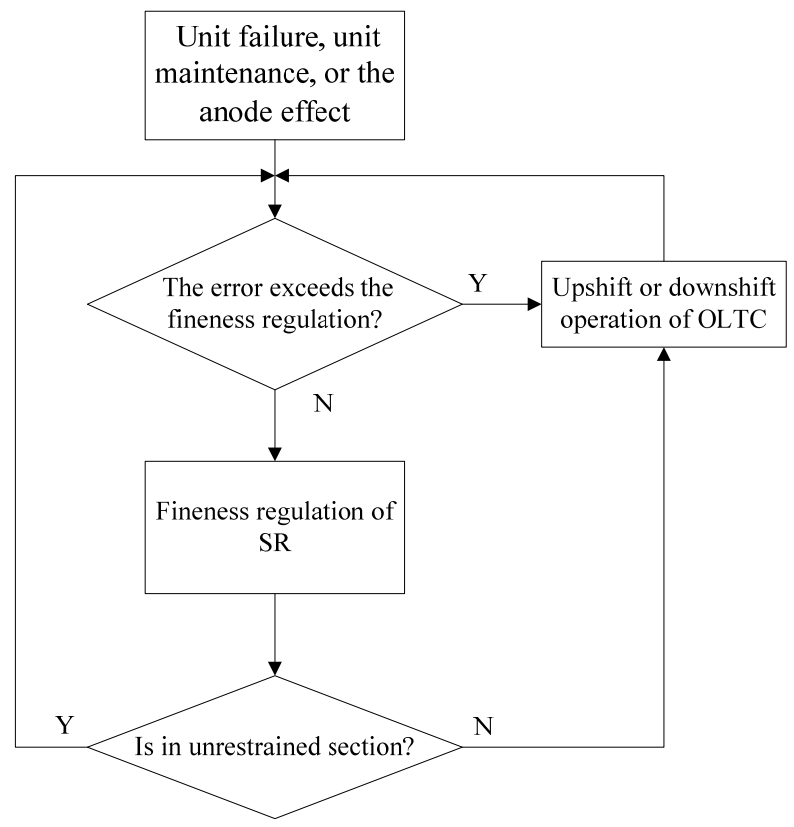

Figure 2. Flowchart of current stabilization control in an aluminum smelter.

taken place to improve the error of the series current.

\section{Simulation Example}

The simulation case consists of an infinite power supply, transmission line, OLTC transformer, and the diode rectifier group with 12 pulses. The system frequency is $50 \mathrm{~Hz}$, the voltage of power supply is $220 \mathrm{kV}$, the length of transmission line is $90 \mathrm{~m}$, and the rated capacity of the OLTC transformers is 250 MVA.

The back electromotive-force of each electrolytic cell is supposed as $1.7 \mathrm{~V}$ [12], then the load consists of 256 electrolytic bathes in series is equivalent to a $0.4352 \mathrm{kV}$ $\mathrm{dc}$ voltage source in series with a variable resistance, that is in the range of $[0.0021083,0.00238099] \Omega$. A choke inductor, about $0.001 \mathrm{H}$, joins up in series to the dc side to reduce the fluctuation. The total case is implemented in the PSCAD/EMTDC, and the model is shown in Figure 5.

Suppose there are 3 anode effects occur simultaneously, the electrolytic series current fluctuation curves with or without the coordinate current stabilization control are shown in Figure 6.

It is easy to recognize that the series current is terrible without any control or only with the control on SR. The reason is that the series current fluctuates seriously when there are 3 anode effects occur at the same time, while the regulation capacity of SR is limit. The OLTC can change discretely the series current significantly. The proposed coordinate control strategy could ensure the adjustment range by means of OLTC, and the precision by means of SR. 


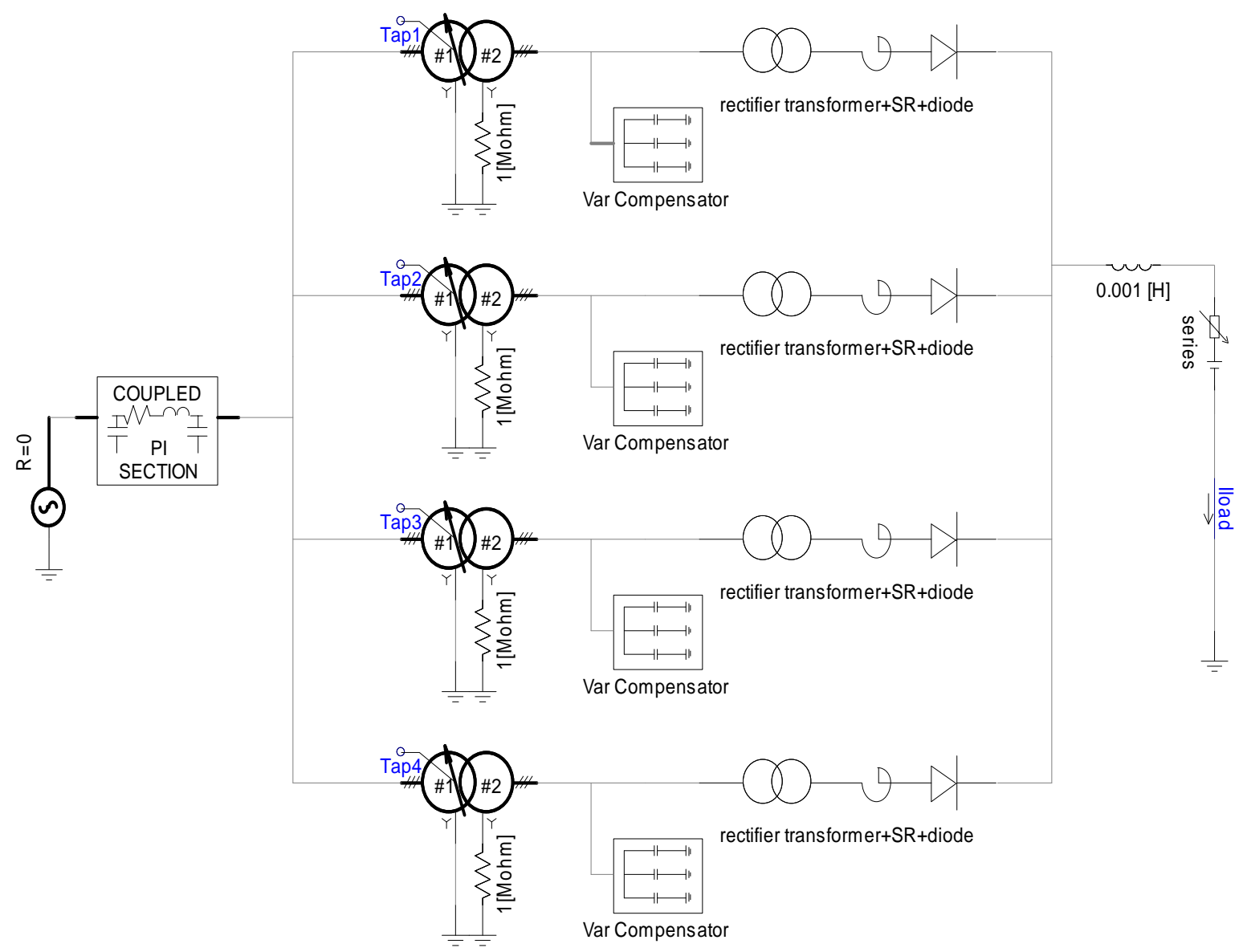

Figure 3. The model of simulation case in PSCAD/EMTDC.

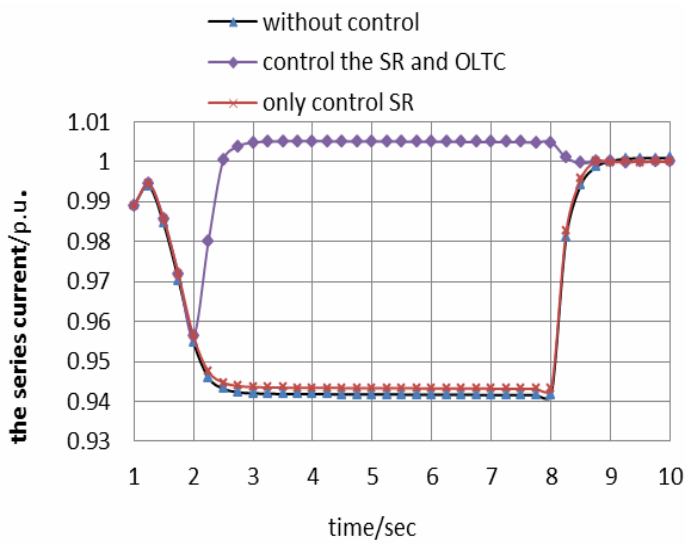

Figure 6. Electrolytic series current fluctuation curve.

\section{Conclusions}

A coordinate current stabilization control in an aluminum smelt is proposed in this paper. The control precision is guaranteed via the close loop control of SR, and the regulation range is large enough with OLTC transformer included. The proposed control strategy is applied into the diode rectifier system, and the effectiveness is verified by a simulation case.

\section{REFERENCES}

[1] C. P. Arnold, K. S. Turner and J. Arrillaga, "Modelling Rectifier Loads for a Multi-machine Transient Stability Programme," IEEE Transactions on Power Apparatus and Systems, Vol. PAS-99, No. 1, 1980, pp. 78-85. doi:10.1109/TPAS.1980.319611

[2] A. Molina-Garciá, M. Kessler, M. C. Bueso, J. A. Fuentes, E. Gómez-Lázaro and F. Faura, "Modeling Aluminum Smelter Plants Using Sliced Inverse Regression with a View Towards Load Flexibility," IEEE Transactions on Power Systems, Vol. 26, No. 1, 2011, pp. 282-293.

[3] A. K. Malaviya and G. A. Bundell, "An Intelligent Controller for Aluminum Smelter Potlines," IEEE Transactions on Industry Applications, Vol. 37, No. 3, 2001, pp. 792-805. doi:10.1109/28.924761

[4] A. Molina, A. Gabaldon, F. Faura and J. A. Fuentes, "New Approaches to Model Electric Demand in Aluminium Smelter Industry," Proceedings of the 36th Annual Industry Application Conference, Chicago, 30 September - 4 October 2001, pp. 1426-1431.

[5] F. J. L. Bindon, "Aluminium and Energy," Power Engineering Journal, Vol. 1, No. 5, 1987, pp. 275-282. doi:10.1049/pe: 19870051

[6] D. B. Corbyn, "D.C. Power Control for Aluminium and Electrolytic Loads," Proceedings of the Institution of 
Electrical Engineers, Vol. 115, No. 11, 1968, pp. 1693-1704. doi:10.1049/piee.1968.0295

[7] A. P. Agalgaonkar, K. M. Muttaqi and S. Perera, "Response Analysis of Saturable Reactors and Tap Changer in an Aluminium Smelting Plant," Proceedings of the 3rd International Conference on Power Systems, Kharagpur, India, 2009.

[8] J. L. Aguero, M. Beroqui and S. Achilles, "Aluminum Plant Load Modeling for Stability Studies," Proceedings of the IEEE Power Engineering Society Summer Meeting, Edmonton, Canada, 18-22 July, 1999, Vol. 2, pp. 13301335.

[9] S. N. Talukdar, J. K. Dickson, R. C. Dugan, M. J. Sprinzen and C. J. Lenda, "On Modeling Transformer and Reactor Saturation Characteristics for Digital and Analog Studies," IEEE Transactions on Power Apparatus and
Systems, Vol. 94, No. 2, 1975, pp. 612-621. doi:10.1109/T-PAS.1975.31889

[10] A. P. Agalgaonkar, K. M. Muttaqi and S. Perera, "Open Loop Response Characterisation of an Aluminium Smelting Plant for Short Time Interval Feeding," Proceedings of the IEEE Power and Energy Society General Meeting, Calgary, Canada, 26-30 July, 2009.

[11] S. B. Abbott, D. A. Robinson, S. Perera, F. A. Darmann, C. J. Hawley and T. P. Beales, "Simulation of HTS Saturable Core-type FCLs for MV Distribution Systems," IEEE Transactions on Power Delivery, Vol. 21, No. 2, 2006, pp. 1013-1018. doi:10.1109/TPWRD.2005.859300

[12] K. Grjotheim and B. J. Welch, "Aluminium Smelter Technology - A Pure and Applied Approach," $2^{\text {nd }}$ Edition, Dusseldorf: Aluminium Verlag, 1987. 